\title{
Automatic Monitoring and Controlling of Weather Condition using Big Data Analytics
}

\author{
I. Karthika ${ }^{1}$, K.P. Porkodi ${ }^{2}$ \\ Assistant Professor, Department of CSE, M. Kumarasamy College of Engineering, Karur, Tamil Nadu, India ${ }^{1}$ \\ Research Scholar, Department of CSE, Al-Ameen Engineering College, India ${ }^{2}$
}

\begin{abstract}
Objective: To monitor and control the weather condition of the state, by scrutinizing each home's weather condition. Analysis: Data which is collected from the sensor is send to the spark analytical streaming for processing; home which is not under the control of normal temperature is update in graph, which is shown by using Thing span, control abnormal temperature by using HVAC system. Findings: In existing system weather condition is only monitored automatically and controlled manually, more energy consumption, whereas in proposed system, sensor receives data and stream data process using spark a, historical data process using Apache hadoop. Improvement: Advance big data analytics tool is used for processing data and automatically controls the weather condition by using HVAC system.
\end{abstract}

Keywords: Apache spark, Apache hadoop, HVAC, Thing Span, Sensor.

\section{INTRODUCTION}

Many countries moving towards the concept of smart city. information [3]. Energy usage is minimized using Making our city as smarter one can be achieved by using combining the user habits and weather forecasts. IOT and advanced big data analytics. All government and Temperature changes occur in home is monitored by private sectors of the city are to be digitized. Digitization Thing span; it is distributed graph platform for analytics. reduces make human works. Internet of things playing a When there is change in occupants increases or decreases major role in smart cities, because it is a main of source of the thermostat. But it is more energy consuming. The information, whereas big data analytics process the data HVAC system, takes only short time to make situation which are received from sensors. Because it creates huge under control. It change to cool or heat accordingly to the information's with high speed. In this paper weather external temperature. Apache yarn used for monitoring the condition of home is monitored by sensors, it generates services and workflow [5]. Spark Machine learning -Mlib huge volume and high speed data, which is normally analysis the pattern of weather and response, it increases cannot be stored and processed by traditional machine, so the efficiency of the system.

advanced big data analytics is used to process the data. Historical data and streaming data stored and processed using Apache hadoop and spark. HVAC system automates controlling process of weather condition of the environment.

\section{EXISTING SYSTEM}

In existing weather condition of the environment is monitored by Embedded system, It uses microprocessor and micro -controller for collecting data from sensor and it send to the Labview using serial communication. Only weather condition is monitored and updated. Further weather controlling process done manually, there is automation process for controlling the weather condition of the environment.

\section{PROPOSED SYSTEM}

Predicting the temperature of a home for making high performance energy management. Home thermostats is used for supplying information to predictive control system. Spark is capable of processing high velocity

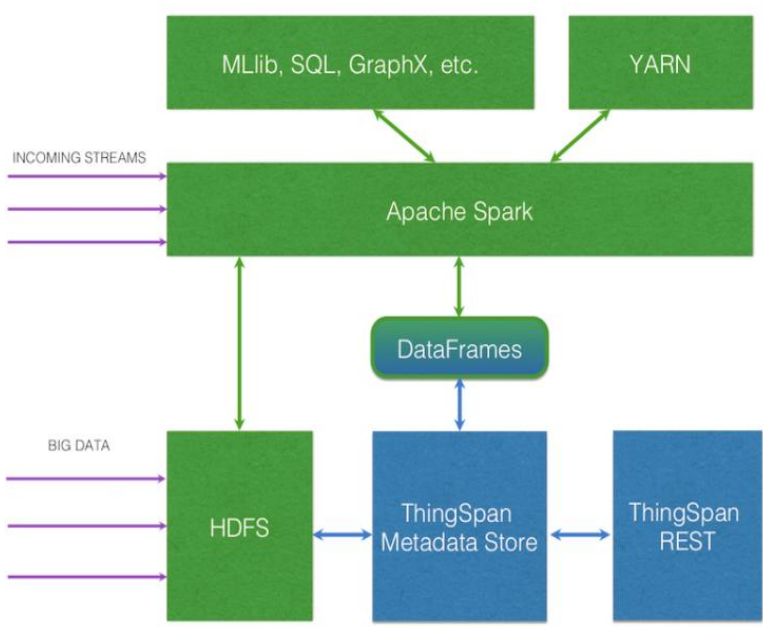

Figure1. Proposed Architecture

\section{DATA LOADING}

Initial data such as state, city, weather forecast, home number and device to be monitored, are stored in Apache 
hadoop. Apache hadoop is capable of storing all kinds and huge volume of data [3]. By using Thing Span RESTAPI these data send to Thing span. The result is shown as an graph below.

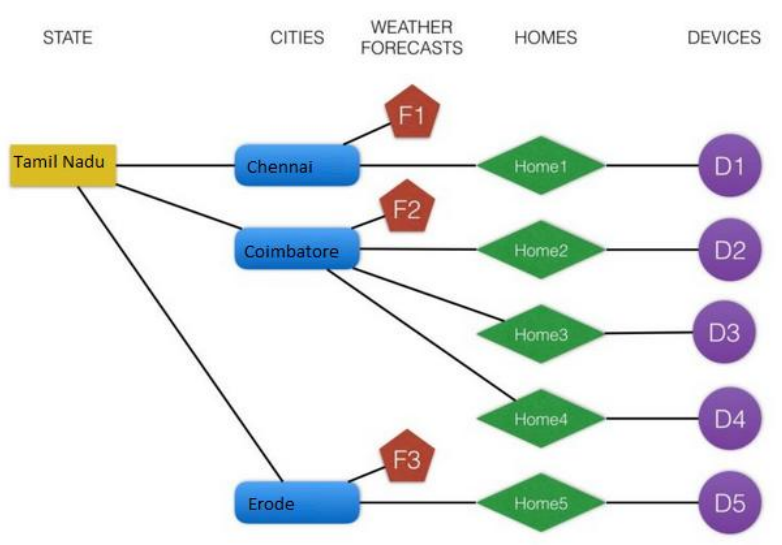

Figure2. Data loading phase

\section{DATA PROCESSING}

Temperature all devices are monitored by sensor. Spark is used for processing incoming temperature from sensor [6]. Spark streaming Engine process the data using RDD transformation. On this transformation, we can identify cities which deviates from the normal temperature. On our scenario city Coimbatore is deviated from temperature. In that home 2 and home 3 needs an immediate activation of thermostat for heating to avoid the cold will arrive shortly. In home2 HVAC want to run for 15 minutes to attain normal temperature. Whereas in home 4 needs to runs for 20 minutes from now.

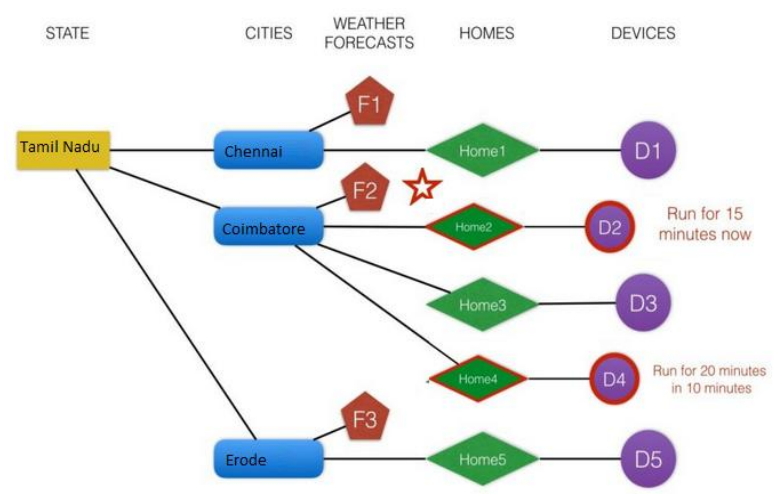

Figure3. Data processing Phase

\section{DATA PREDICTION AND ACTION}

This information will be provided to HVAC thermostat controller for activation. It reduces the sudden change in temperature. It takes only minimum time to heat or cool the environment. So it keep hot in winter and cool in summer. Its main advantage is saving energy, Electricity usage is reduced. Thing span is powerful graph analytics tool. Real-time weather of the whole state monitored in the form of graph regularly [6].

\section{CONCLUSION}

Smart home embed with the sensors and advance analytical software. In our system, weather condition of whole state is monitored and controlled by using hvac system. Weather condition monitored as graph using thing span. Sensors send its weather report to spark for real-time data analytics. Processing data exceeds normal temperature of weather, HVAC system is activated it reduces the weather condition to normal temperature.

\section{REFERENCES}

[1] P.Susmitha and G.Sowmyabala "Design and Implementation of Weather Monitoring and Controlling System" International Journal of Computer Applications Volume 97-No.3, July 2014.

[2] Kang. J. and Park S. "Integrated comfort sensing system on indoor climate" Sensors and Actuators. 2000. 302-307.

[3] Thilagamani, S. and N. Shanthi, 2010. Literature survey on enhancing cluster quality. Int. J. Comput. Sci. Eng.., 2: 1999-2002.

[4] S. Chitra, B. Madhusudhanan, G. Sakthidharan, P. Saravanan, Local Minima Jump PSO for Workflow Scheduling in Cloud Computing Environments, Springer, ISBN 364241673X, 12251234, 2014.

[5] E.T. Venkatesh , P. Thangaraj, and S. Chitra , " An Improved Neural Approach for Malignant and Normal Colon Tissue Classification from Oligonucleotide Arrays ," European J. Scientific Research, vol. 54 , pp. $159-164,2011$

[6] S Saravanan, V Venkatachalam,"Advance Map Reduce Task Scheduling algorithm using mobile cloud multimedia services architecture" IEEE Digital Explore,pp21-25,2014.

[7] Eiman Al Nuaimi, Hind Al Neyadi, Nader MohamedEmail author and Jameela Al-Jaroodi "Applications of big data to smart cities" Journal of Internet Services and Applications DOI: 10.1186/s13174-015-0041-5

[8] IBM Software Information Management "Managing big data for smart grids and smart meters"IMW14628-USEN-00

[9] S Saravanan, V Venkatachalam, "Enhanced bosa for implementing map reduce task scheduling algorithm" International Journal of Applied Engineering Research, Vol 10(85),pp60-65,2015.

[10] Muthulakshmi A, Dr. S. Baghavathi Priya "A survey on weather forecasting to predict rainfall using big data analytics." International Journal of Innovative Science, Engineering \& Technology, Vol. 2 Issue 10, October2015.

[11] Walid Mohamed Alyand Mohamed Kholief "Intelligent Cloud Based Decision Support Framework for Smart Grid" International Journal of Computer and Information Technology (ISSN: 2279 0764) Volume 05 -Issue 01, January 2016 